PROCEEDINGS OF THE AMERICAN MATHEMATICAL SOCIETY

Volume 124, Number 8, August 1996

\title{
ENDOMORPHISM RINGS OF COMPLETELY PURE-INJECTIVE MODULES
}

\author{
JOSÉ L. GÓMEZ PARDO AND PEDRO A. GUIL ASENSIO
}

(Communicated by Ken Goodearl)

\begin{abstract}
Let $R$ be a ring, $E=E\left(R_{R}\right)$ its injective envelope, $S=\operatorname{End}\left(E_{R}\right)$ and $J$ the Jacobson radical of $S$. It is shown that if every finitely generated submodule of $E$ embeds in a finitely presented module of projective dimension $\leq 1$, then every finitley generated right $S / J$-module $X$ is canonically isomorphic to $\operatorname{Hom}_{R}\left(E, X \otimes_{S} E\right)$. This fact, together with a well-known theorem of Osofsky, allows us to prove that if, moreover, $E / J E$ is completely pure-injective (a property that holds, for example, when the right pure global dimension of $R$ is $\leq 1$ and hence when $R$ is a countable ring), then $S$ is semiperfect and $R_{R}$ is finite-dimensional. We obtain several applications and a characterization of right hereditary right noetherian rings.
\end{abstract}

\section{INTRODUCTION}

Let $R$ be a ring, $M_{R}$ a right $R$-module, and $S=\operatorname{End}\left(M_{R}\right)$. Then there exists an adjoint pair:

$$
\operatorname{Hom}_{R}(M,-): \operatorname{Mod}-R \leftrightarrows \operatorname{Mod}-S:-\otimes_{S} M
$$

which induces a functorial morphism $\alpha: 1_{\operatorname{Mod}-S} \rightarrow \operatorname{Hom}_{R}\left(M,-\otimes_{S} M\right)$. If $X$ is a right $S$-module such that $\alpha_{X}$ is an isomorphism, we will say that $X_{S}$ is $M$ invariant. It is well known that when every right $S$-module $X$ is $M$-invariant, useful information can be passed from $M_{R}$ to $S$. This is what happens, for example, when $M_{R}$ is a finitely generated projective module, which makes it possible to characterize properties of the endomorphism ring $S$ in terms of $M_{R}$. This property also holds when $M_{R}$ is finitely presented and $S$ is a (von Neumann) regular ring and this, coupled with Osofsky's theorem $[8,9]$ that asserts that a ring whose cyclic right modules are all injective is semisimple, has been exploited in [3] to obtain an easy proof of the result of Damiano that shows that a right PCI ring (i.e., a ring with each proper cyclic right module injective) is right noetherian.

This technique was also (implicitly) applied in [1] to a right hereditary ring $R$ whose injective envelope $E\left(R_{R}\right)$ is projective, showing that $R$ is, in this case, a (two-sided) hereditary artinian QF-3 ring. An extension in [3, Corollary 6] shows that if $E\left(R_{R}\right)$ is just finitely presented (instead of projective), then $R$ is a right

Received by the editors June 23, 1994 and, in revised form, October 5, 1994 and November 29, 1994.

1991 Mathematics Subject Classification. Primary 16S50; Secondary 16D50, 16E60, 16P60, $16 \mathrm{~S} 90$.

Work partially supported by the DGICYT (PB93-0515, Spain). The first author was also partially supported by the European Community (Contract CHRX-CT93-0091). 
artinian ring with Morita duality. The key point of this proof is to show that $R$ is right finite-dimensional. But, as the endomorphism ring $S$ of $E=E\left(R_{R}\right)$ is regular, all the cyclic right $S$-modules are $E$-invariant. This makes it possible to transfer the injectivity property and then to use Osofsky's theorem to show that $S$ is semisimple.

In this paper we consider the rather more general situation that arises when the injective envelope $E_{R}=E\left(R_{R}\right)$ of a ring $R$ has the property that every finitely generated submodule embeds in a finitely presented module whose projective dimension is $\leq 1$ (this includes the right hereditary rings with finitely presented injective envelope, but also the rings $R$ such that every finitely generated submodule of $E_{R}$ embeds in a free module). If $S=\operatorname{End}\left(E_{R}\right)$ and $J$ is the radical of $S$, we prove in Theorem 1.6 that each finitely generated right $S / J$-module is $E$-invariant-a result that will be our main tool in the rest of the paper. This allows us to apply the transfer techniques sketched above to the ring $S / J$ and hence substantially broaden the scope of these methods. In this setting, we usually cannot expect that the endomorphism ring $S$ is semisimple. In general, it is not even regular. However, we show that when certain quotients of $E_{R}$ are pure-injective, then $S$ is semiperfect and hence $R_{R}$ is finite-dimensional. More specifically, we assume that $E / J E$ is a completely pure-injective $R$-module, i.e., a module such that each pure quotient of itself is pure-injective. We give several applications and we extend [3, Corollary 6] by proving that if $R$ is right hereditary and every finitely generated submodule of $E_{R}$ is finitely presented, then $R$ is right noetherian.

In the last part of the paper we consider rings $R$ whose right pure global dimension (cf. $[6,7]$ ) is $\leq 1$. This includes all countable rings. If every finitely generated submodule of $E_{R}$ embeds in a finitely presented module of projective dimension $\leq 1$, then we show that $E / J E$ is pure-injective (Theorem 2.1 ), so that $E / J E$ is completely pure-injective in this case and hence $R$ is, again, finite-dimensional. As an application we show that, for these rings, the property that $R$ is right nonsingular and every finitely generated right $R$-module embeds in a free module is right-left symmetric.

We refer to [5] and [11] for all undefined notions used in the text.

\section{M-INVARIANT MODULES}

Let ${ }_{S} M_{R}$ be a bimodule. We have a pair or adjoint functors $\operatorname{Hom}_{R}(M,-)$ : $\operatorname{Mod}-R \leftrightarrows \operatorname{Mod}-S:-\otimes_{S} M$ and the corresponding adjunction morphisms $\alpha_{X}$, for every $X \in \operatorname{Mod}-S$. The right $S$-modules $X$ such that $\alpha_{X}$ is an isomorphism will, again, be called $M$-invariant. The following result is well known (cf. [12], [11]).

Proposition 1.1. Let ${ }_{S} M_{R}$ be a bimodule. Then the following assertions hold:

(i) If $L_{R}$ is pure-injective, then $\operatorname{Hom}_{R}(M, L)$ is a pure-injective right $S$-module.

(ii) If ${ }_{S} M$ is flat and $L_{R}$ is $M$-injective, then $\operatorname{Hom}_{R}(M, L)$ is injective.

Our interest in $M$-invariant modules is motivated by the fact that certain injectivity properties are easily transferred to these modules. From Proposition 1.1 we have:

Proposition 1.2. Let ${ }_{S} M_{R}$ be a bimodule and $X$ an $M$-invariant right $S$-module. Then the following assertions hold:

(i) If $X \otimes_{S} M$ is pure-injective, then $X$ is pure-injective.

(ii) If ${ }_{S} M$ is flat and $X \otimes_{S} M$ is $M$-injective, then $X$ is injective. 
In order to exploit Proposition 1.2 we need to have $M$-invariant $S$-modules. Recall that if $E_{R}$ is (quasi-)injective (or pure-injective), then $S / J$ (where $S=$ $\operatorname{End}\left(E_{R}\right)$ and $\left.J=J(S)\right)$ is a regular ring and idempotents lift modulo $J$. We want to apply Osofsky's theorem to $S / J$ and for this we need to prove that the cyclic right $S / J$-modules are $E$-invariant. We start by giving a useful sufficient condition for $\alpha_{X}$ to be a monomorphism.

Proposition 1.3. Let $P_{R}$ be a finitely generated projective module, $E=E\left(P_{R}\right)$ and $S=\operatorname{End}\left(E_{R}\right)$. Then $\alpha_{X}$ is a monomorphism for each finitely generated right $S / J$-module $X$.

Proof. Since $X$ is an $S / J$-module and $X J=0$, we have a free presentation of $X$ in Mod- $S$, say $S^{(I) \stackrel{h}{\longrightarrow}} S^{n} \stackrel{p}{\rightarrow} X \rightarrow 0$, where $J^{n}=J\left(S^{n}\right) \subseteq \operatorname{Ker} p=\operatorname{Im} h$. Applying $-\otimes_{S} E$ we obtain an exact sequence in Mod- $R$

$$
E^{(I)} \stackrel{h_{*}}{\longrightarrow} E^{n} \stackrel{p_{*}}{\longrightarrow} X \otimes_{S} E \rightarrow 0
$$

Let $Z:=\operatorname{Im} h_{*}=\operatorname{Ker} p_{*}$, with canonical projection $v: E^{(I)} \rightarrow Z$ and canonical injection $u: Z \rightarrow E^{n}$. Then each $f \in \operatorname{Hom}_{R}\left(E, E^{n}\right)$ such that $p_{*} \circ f=0$ factors in the form $f=u \circ f^{\prime}$, where $f^{\prime} \in \operatorname{Hom}_{R}(E, Z)$. Since $P$ is projective, we obtain a morphism $g: P \rightarrow E^{(I)}$ that makes the diagram

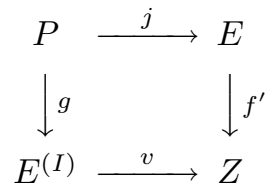

commute, where $j$ is the canonical inclusion. Since $P$ is finitely generated, $g(P) \subseteq$ $E^{(F)}$ for some finite subset $F$ of $I$. As $E$ is injective, there exists a homomorphism $t: E \rightarrow E^{(I)}$ such that $t \circ j=g$. Hence $h_{*} \circ t \circ j=h_{*} \circ g=f \circ j$, so that $\left(h_{*} \circ t-f\right) \circ j=0$. Since $j$ is an essential monomorphism by hypothesis, $\operatorname{Ker}\left(h_{*} \circ t-f\right)$ is essential in $E$. Consider the following commutative diagram of right $S$-modules:

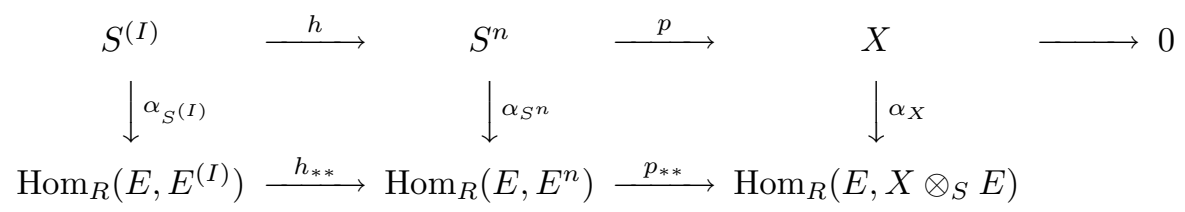

Then $f \in \operatorname{Hom}_{R}\left(E, E^{n}\right)$ and $f \in \operatorname{Ker} p_{* *}$, so there exists $t \in \operatorname{Hom}_{R}\left(E, E^{(I)}\right)$ such that $h_{* *}(t)-f$ has essential kernel and, hence, belongs to $J(S)^{n}$. Thus $h_{* *}(t)-f \in$ $\alpha_{S^{n}}(\operatorname{Ker} p)$. On the other hand, since $\operatorname{Im} t \subseteq E^{(F)}$ for $F$ finite, there exists $q \in S^{(I)}$ such that $t=\alpha_{S^{(I)}}(q)$ and so $h_{* *}(t)=\left(\alpha_{S^{n}} \circ h\right)(q) \in \alpha_{S^{n}}(\operatorname{Ker} p)$. Thus we have that $f \in \alpha_{S^{n}}(\operatorname{Ker} p)$ and this implies that $\alpha_{X}$ is a monomorphism.

Recall that $R$ is called a right Kasch ring whenever $E\left(R_{R}\right)$ is a cogenerator of Mod- $R$. From the preceding result we immediately obtain:

Corollary 1.4. Let $R$ be a right Kasch ring. Then $\operatorname{End}\left(E\left(R_{R}\right)\right)$ is also a right Kasch ring.

Proof. Let $E=E\left(R_{R}\right), S=\operatorname{End}\left(E_{R}\right)$ and $J=J(S)$. If $C$ is a simple right $S$ module, then $C J=0$ and so $C$ is an $S / J$-module. Thus $\alpha_{C}$ is a monomorphism by 
Proposition 1.3 and, as $C \otimes_{S} E$ is cogenerated by $E$, we obtain a monomorphism $C \stackrel{\alpha_{C}}{\longrightarrow} \operatorname{Hom}_{R}\left(E, C \otimes_{S} E\right) \rightarrow \operatorname{Hom}_{R}\left(E, E^{I}\right) \cong S^{I}$, for some set $I$. Hence $C$ embeds in $S_{S}$.

Now, in order to obtain $E$-invariant modules from Proposition 1.3, we need to give conditions for $\alpha_{X}$ to be an epimorphism. The following lemma will be crucial for this purpose.

Lemma 1.5. Let $P_{R}$ be a finitely generated projective right $R$-module, $E=E\left(P_{R}\right)$ its injective hull, and $S=\operatorname{End}\left(E_{R}\right)$. Assume that each finitely generated submodule of $E$ embeds in a finitely presented module of projective dimension $\leq 1$. Then, for each finitely generated right $S / J$-module $X, \operatorname{Hom}_{R}\left(E / P, X \otimes_{S} E\right)=0$.

Proof. Let $f \in \operatorname{Hom}_{R}\left(E / P, X \otimes_{S} E\right)$ and $\pi: E \rightarrow E / P$ the canonical projection. We want to prove that $g=f \circ \pi=0$. Since $P$ is finitely generated, $E$ is the direct limit of all its finitely generated submodules that contain $P$. Thus it will be enough to show that if $P \subseteq Z \subseteq E$ and $Z$ is finitely generated, then $g(Z)=0$. By hypothesis, there exists a finitely presented right $R$-module $F$ such that $\operatorname{pd}(F) \leq 1$, and a monomorphism $\varphi: Z \rightarrow F$. Then, regarding $P$ as a submodule of $F$, we get the following commutative diagram:

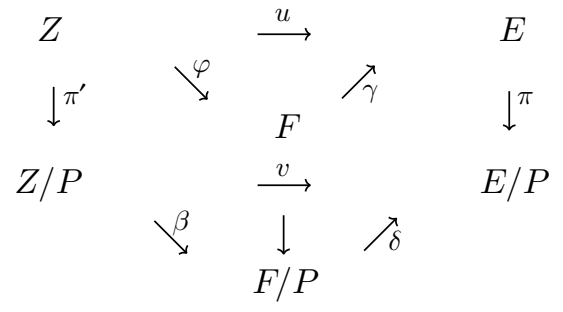

where $\beta$ is the monomorphism induced by $\varphi, \gamma$ is obtained by the injectivity of $E$, and $\delta$ is induced by $\gamma$. We have that $F / P$ is a finitely presented module. Consider the functorial exact sequence

$$
0=\operatorname{Ext}_{R}^{1}(P,-) \rightarrow \operatorname{Ext}_{R}^{2}(F / P,-) \rightarrow \operatorname{Ext}_{R}^{2}(F,-)=0 .
$$

Since $\operatorname{pd}(F) \leq 1$, the last term is zero, and so $\operatorname{pd}(F / P) \leq 1$. Next let $S^{(I)} \rightarrow$ $S^{n} \stackrel{p}{\rightarrow} X \rightarrow 0$ be a free presentation of $X$ in Mod- $S$ and consider the induced exact sequence in Mod- $R, E^{(I)} \rightarrow E^{n} \stackrel{p \otimes E}{\longrightarrow} X \otimes_{S} E \rightarrow 0$. Set $Y=\operatorname{Ker}\left(p \otimes_{S} E\right)$. From the short exact sequence $0 \rightarrow K \rightarrow E^{(I)} \rightarrow Y \rightarrow 0$ we obtain the natural exact sequence

$$
\operatorname{Ext}_{R}^{1}\left(F / P, E^{(I)}\right) \rightarrow \operatorname{Ext}_{R}^{1}(F / P, Y) \rightarrow \operatorname{Ext}_{R}^{2}(F / P, K) .
$$

Since $\operatorname{pd}(F / P) \leq 1$, we have that $\operatorname{Ext}_{R}^{2}(F / P, K)=0$ and, as $F / P$ is finitely presented and $E$ is injective, $\operatorname{Ext}_{R}^{1}\left(F / P, E^{(I)}\right) \cong \operatorname{Ext}_{R}^{1}(F / P, E)^{(I)}=0$. Thus $\operatorname{Ext}_{R}^{1}(F / P, Y)=0$ and so we have an exact sequence

$$
\operatorname{Hom}_{R}\left(F / P, E^{n}\right) \stackrel{(p \otimes E)_{*}}{\longrightarrow} \operatorname{Hom}_{R}(F / P, X \otimes E) \rightarrow \operatorname{Ext}_{R}^{1}(F / P, Y)=0
$$

which shows that $(p \otimes E)_{*}=\operatorname{Hom}_{R}(F / P, p \otimes E)$ is an epimorphism. Hence, there exists a morphism $\epsilon: F / P \rightarrow E^{n}$ such that $f \circ \delta=(p \otimes E) \circ \epsilon$. But, as $E^{n}$ is injective and $v$ is a monomorphism, $\epsilon \circ \beta: Z / P \rightarrow E^{n}$ can be extended to a map $\mu$ : $E / P \rightarrow E^{n}$ such that $\mu \circ v=\epsilon \circ \beta$. This gives $(p \otimes E) \circ \mu \circ v=(p \otimes E) \circ \epsilon \circ \beta=f \circ \delta \circ \beta=$ 
$f \circ v$. Thus we have that $\left.g\right|_{Z}=g \circ u=f \circ \pi \circ u=f \circ v \circ \pi^{\prime}=(p \otimes E) \circ \mu \circ v \circ \pi^{\prime}=$ $(p \otimes E) \circ \mu \circ \pi \circ u$, so that it remains to prove that $(p \otimes E) \circ \mu \circ \pi \circ u=0$.

If $p_{i}: E^{n} \rightarrow E$ are the canonical projections for $i=1, \ldots, n$, then each $p_{i} \circ \mu \circ \pi$ is an element of $S$ whose kernel contains $P$. Therefore $p_{i} \circ \mu \circ \pi \in J(S)$. Now, let $x$ be an element of $E$ and set $e_{i}=\left(\delta_{i j}\right)_{j=1, \ldots, n} \in S$. Since $X J=0$ and $p_{i} \circ \mu \circ \pi \in J$, $((p \otimes E) \circ \mu \circ \pi \circ u)(x)=(p \otimes E)((\mu \circ \pi)(x))=\sum_{i=1}^{n} p\left(e_{i}\right) \otimes\left(p_{i} \circ \mu \circ \pi\right)(x)=$ $\sum_{i=1}^{n} p\left(e_{i}\right) \cdot\left(p_{i} \circ \mu \circ \pi\right) \otimes x=0$. This completes the proof.

Theorem 1.6. Let $P_{R}$ be a finitely generated projective module, $E=E\left(P_{R}\right)$ and $S=\operatorname{End}\left(E_{R}\right)$. Assume that each finitely generated submodule of $E$ embeds in a finitely presented module of projective dimension $\leq 1$. Then each finitely generated right $S / J$-module is E-invariant.

Proof. Let $X$ be a finitely generated right $S / J$-module. By Proposition $1.3 \alpha_{X}$ is a monomorphism. It remains to prove that $\alpha_{X}$ is an epimorphism. Consider a free presentation $S^{(I)} \rightarrow S^{n} \stackrel{p}{\rightarrow} X \rightarrow 0$ of $X$ in Mod-S. Tensoring with ${ }_{S} E$ yields an exact sequence in Mod- $R, E^{(I)} \rightarrow E^{n} \stackrel{p \otimes E}{\longrightarrow} X \otimes_{S} E \rightarrow 0$. Now, if $\varphi \in \operatorname{Hom}_{R}\left(E, X \otimes_{S} E\right)$ and $j: P \rightarrow E$ is the canonical inclusion, there is by the projectivity of $P$ a morphism $t: P \rightarrow E^{n}$ such that $\varphi \circ j=(p \otimes E) \circ t$. Then, as $E$ is injective, there exists $h: E \rightarrow E^{n}$ such that $h \circ j=t$. Thus we have $(p \otimes E) \circ h \circ j=(p \otimes E) \circ t=\varphi \circ j$, so that $(\varphi-(p \otimes E) \circ h) \circ j=0$. Hence $g:=\varphi-(p \otimes E) \circ h$ factors through the projection $\pi: E \rightarrow E / P$, say as $g=f \circ \pi$. By Lemma 1.5 we have that $f=0$, and so $g=0$ and $\varphi=(p \otimes E) \circ h$. Thus we see that $(p \otimes E)_{*}$ is an epimorphism and the commutative diagram:

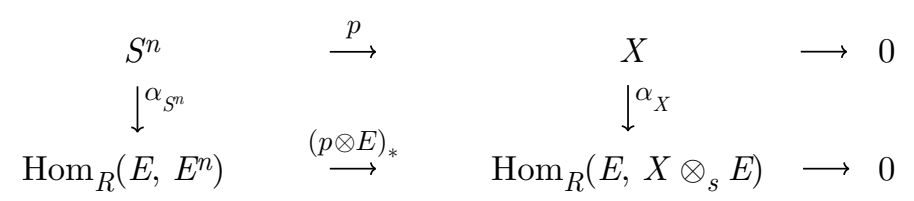

shows that $\alpha_{X}$ is indeed an epimorphism.

If $E_{R}$ is quasi-injective and $S=\operatorname{End}\left(E_{R}\right)$, then $S / J$ is a regular right selfinjective ring. If we set $\bar{E}:=(S / J) \otimes_{S} E=E / J E$, then we have a bimodule ${ }_{S / J} \bar{E}_{R}$ and, if $X \in \operatorname{Mod}-S / J$, we have that

$$
X \otimes_{S} E \cong\left(X \otimes_{S / J} S / J\right) \otimes_{S} E \cong X \otimes_{S / J}\left((S / J) \otimes_{S} E\right) \cong X \otimes_{S / J} \bar{E} .
$$

Thus, if we identify $X \otimes_{S} E$ with $X \otimes_{S / J} \bar{E}$, and if $\bar{\alpha}_{X}: X \rightarrow \operatorname{Hom}_{R}\left(\bar{E}, X \otimes_{S / J} \bar{E}\right)$ is the canonical morphism and $p: E \rightarrow \bar{E}$ the canonical projection, we see that $\operatorname{Hom}_{R}\left(p, X \otimes_{S} E\right) \circ \bar{\alpha}_{X}=\alpha_{X}$. Since $\operatorname{Hom}_{R}\left(p, X \otimes_{S} E\right)$ is a monomorphism, if $X_{S}$ is $E$-invariant, then $X_{S / J}$ is $\bar{E}$-invariant.

Specifically, if $X=S / J$, then we have proved

Corollary 1.7. Let $P_{R}$ be a finitely generated projective module, $E=E\left(P_{R}\right)$, $S=\operatorname{End}\left(E_{R}\right)$ and $J=J(S)$. If every finitely generated submodule of $E$ embeds in a finitely presented module of projective dimension $\leq 1$, there is a canonical isomorphism $S / J=\operatorname{End}(E / J E)$.

Proposition 1.8. Let $E_{R}$ be quasi-injective (or pure-injective) and let $X$ be a right $S / J$-module which is E-invariant. If $X \otimes_{S} E$ is either E-injective or pure-injective, then $X_{S / J}$ is injective. 
Proof. Let $\bar{E}=E / J E$. Since $X$ is $E$-invariant, it is also $\bar{E}$-invariant. On the other hand, as $S / J$ is regular, $S / J \bar{E}$ is flat. By Proposition 1.2 applied to the adjunction defined by ${ }_{S / J} \bar{E}_{R}$, if we assume that $X \otimes_{S} E \cong X \otimes_{S / J} \bar{E}$ is $E$-injective, we get that $X_{S / J}$ is injective. Similarly, if $X \otimes_{S / J} \bar{E}$ is pure-injective, then $X_{S / J}$ is pure-injective and hence, since $S / J$ is regular, injective.

We will say that a module $M$ is completely pure-injective when every pure quotient of $M$ is pure-injective. (Note the change of terminology with respect to [3].)

Corollary 1.9. Let $P_{R}$ be a finitely generated projective module, $E=E\left(P_{R}\right), S=$ $\operatorname{End}\left(E_{R}\right)$, and $J=J(S)$. Assume that every finitely generated submodule of $E_{R}$ embeds in a finitely presented right $R$-module of projective dimension $\leq 1$ and that $E / J E$ is completely pure-injective. Then $S$ is semiperfect and $P_{R}$ is finitedimensional.

Proof. By Theorem 1.6, each finitely generated right $S / J$-module $X$ is $E$-invariant. Since the canonical projection $S / J \rightarrow X$ is a pure epimorphism (since $S / J$ is regular), we have that the induced $R$-epimorphism $E / J E \rightarrow X \otimes_{S} E$ is also pure. Thus $X \otimes_{S} E$ is a pure-injective right $R$-module by hypothesis, and by Proposition $1.8, X_{S / J}$ is injective. Then, by Osofsky's theorem $[8,9], S / J$ is semisimple and hence $S$ is semiperfect. This is equivalent to $E_{R}$ (and hence to $P_{R}$ ) being finitedimensional.

The preceding corollary can be regarded as a generalization of [3, Corollary 6]. A more specific extension of this result is the following:

Corollary 1.10. Let $R$ be a right hereditary ring. Then $R$ is right noetherian if and only if every finitely generated submodule of $E\left(R_{R}\right)$ is finitely presented.

Proof. If every finitely generated submodule of $E\left(R_{R}\right)$ is finitely presented, then $R_{R}$ is right finite-dimensional by Corollary 1.9. Thus, using [5, Corollary 5.20], we see that $R$ is right noetherian. The converse is clear.

\section{Rings of PURE GLOBAL DIMENSION LESS THAN OR EQUAL TO ONE}

Recall that the pure-injective dimension of a right $R$-module $M$ is defined as the smallest nonnegative integer (or $\infty$ ) such that there exists an exact sequence $0 \rightarrow M \rightarrow E_{0} \rightarrow E_{1} \rightarrow \cdots \rightarrow E_{n} \rightarrow 0$, where the $E_{i}, i=0, \ldots, n$, are pure-injective modules and the associated short exact sequences are pure exact. The supremum of the pure-injective dimensions of the right $R$-modules is called the right pure global dimension of $R[7,6]$, and is denoted by r. $\operatorname{pgldim}(R)$. Thus the rings $R$ such that r. $\operatorname{pgldim}(R) \leq 1$ provide a natural source of completely pure-injective modules. The following theorem will be useful in order to apply our results to these rings.

Theorem 2.1. Let $R$ be a ring, $E=E\left(R_{R}\right), S=\operatorname{End}\left(E_{R}\right)$ and $J=J(S)$. If every finitely generated submodule of $E_{R}$ embeds in a finitely presented module of projective dimension $\leq 1$, then $E / J E$ is a pure-injective $R$-module.

Proof. Let $\bar{E}=E / J E$. Consider the exact sequence in Mod- $R, 0 \rightarrow R \stackrel{j}{\rightarrow}$ $E \rightarrow E / R \rightarrow 0$, and let $g \in \operatorname{Hom}_{R}(R, \bar{E}) \cong \bar{E}$. Then $g$ induces a homomorphism $h: R_{R} \rightarrow E$ such that if $q: E \rightarrow \bar{E}$ is the canonical projection, then 
$q \circ h=g$. By the injectivity of $E, h$ extends to $t: E \rightarrow E$, so $g$ extends to a morphism $q \circ t: E \rightarrow \bar{E}$. Thus, in the exact sequence

$$
\operatorname{Hom}_{R}(E / R, \bar{E}) \rightarrow \operatorname{Hom}_{R}(E, \bar{E}) \stackrel{j_{*}}{\rightarrow} \operatorname{Hom}_{R}(R, \bar{E}),
$$

$j_{*}$ is an epimorphism and hence an isomorphism since $\operatorname{Hom}_{R}(E / R, \bar{E})=0$ by Lemma 1.5. Since $S / J$ is $E$-invariant by Theorem 1.6, we have isomorphisms of left $S / J$-modules:

$$
\bar{E} \cong \operatorname{Hom}_{R}(E, \bar{E}) \cong \operatorname{Hom}_{R}\left(E,(S / J) \otimes_{S} E\right) \cong S / J .
$$

Let $\bar{E}^{*}=\operatorname{Hom}_{S / J}(\bar{E}, S / J)$. Since $\bar{E}$ is reflexive as a $S / J$-module,

$$
\bar{E} \cong \operatorname{Hom}_{S / J}\left(\bar{E}^{*}, S / J\right) \text {. }
$$

Since $S / J$ is right self-injective, applying Proposition 1.1 to the bimodule ${ }_{R} \bar{E}_{S / J}^{*}$ we obtain that $\bar{E}$ is a pure-injective right $R$-module.

Remark. As a consequence of Theorem 2.1 we see that, in Corollary 1.9, it is enough to assume that every proper pure quotient of $E / J E$ is pure-injective, instead of requiring that $E / J E$ be completely pure-injective.

Corollary 2.2. Let $R$ be a ring such that $\mathrm{r}$. $\operatorname{pgldim}(R) \leq 1$. Assume, further, that every finitely generated submodule of $E\left(R_{R}\right)$ embeds in a finitely presented module of projective dimension $\leq 1$. Then $R$ is right finite-dimensional.

Proof. If $E=E\left(R_{R}\right)$ we have, by Theorem 2.1, that $E / J E$ is pure-injective and hence completely pure-injective. Then $R$ is right finite-dimensional by Corollary 1.9 .

An interesting class of rings of right pure global dimension $\leq 1$ is the class of countable rings $[6,7]$. For instance, it follows from the preceding results that every countable ring $R$ such that every finitely generated submodule of $E\left(R_{R}\right)$ embeds in a finitely presented module of projective dimesion $\leq 1$ is finite-dimensional.

The following result is a partial generalization of [1, Theorem 3.2], and shows that the rings such that r. $\operatorname{pgldim}(R) \leq 1$ and $E\left(R_{R}\right)$ is projective are not far from being right $\mathrm{QF}-3$ rings (but they need not be, as the ring $R=\left(\begin{array}{c}\mathbb{Q} \mathbb{Q} \\ 0\end{array}\right)$ shows).

Corollary 2.3. Let $R$ be a ring such that r. $\operatorname{pgldim}(R) \leq 1$ and $E\left(R_{R}\right)$ is projective. Then $R$ has a faithful injective right ideal.

Proof. By Corollary $2.2 R$ is right finite-dimensional and, using [10, Lemma 2], we obtain the result.

The rings $R$ such that every finitely generated right $R$-module embeds in a free module have been called right FGF by Faith [2]. It is still an open problem whether a right $\mathrm{FGF}$ ring must be $\mathrm{QF}$.

Corollary 2.4. Let $R$ be a right FGF ring such that $\mathrm{r}$. $\operatorname{pgldim}(R) \leq 1$ and $R$ has essential right socle. Then $R$ is $Q F$.

Proof. $R$ is right finite-dimensional by Corollary 2.2. Thus $\operatorname{Soc}\left(R_{R}\right)$ is finitely generated and, as $R_{R}$ has essential socle, we see that $R_{R}$ has finite essential socle. Since each finitely generated right module embeds in a (finitely generated) free right $R$-module, we see that every finitely generated right module has finite essential socle, so that $R$ is right artinian. Then $R$ is QF by [2]. 
Recall that a ring homomorphism $\varphi: R \rightarrow Q$ is a right flat epimorphism of rings (or a perfect right localization of $R$ ) precisely when ${ }_{R} Q$ is flat and the canonical morphism $Q \otimes_{R} Q \rightarrow Q$ is an isomorphism. Goodearl proved that if $Q$ is the right maximal quotient ring of a right nonsingular ring $R$, then the canonical morphism $R \rightarrow Q$ is a left flat epimorphism if and only if every finitely generated nonsingular right $R$-module embeds in a free module [4, Theorem 7]. In general, this condition is not right-left symmetric, as is shown by the endomorphism ring of an infinitedimensional vector space over a field. However, if $\mathrm{r}$. $\operatorname{ggldim}(R) \leq 1$, then we have symmetry.

Corollary 2.5. Let $R$ be a ring such that $\mathrm{r} \cdot \operatorname{pgldim}(R) \leq 1$. Then the following conditions are equivalent:

(i) $R$ is right nonsingular and every finitely generated nonsingular right $R$-module embeds in a free module.

(ii) $R$ is left nonsingular and every finitely generated nonsingular left $R$-module embeds in a free module.

(iii) $R$ has a semisimple two-sided maximal quotient ring.

Proof. (i) $\Rightarrow$ (iii) Let $Q=Q_{\max }^{r}(R)$ be the maximal right quotient ring of $R$. By Corollary 2.2, $R$ is right finite-dimensional and so $Q$ is semisimple [11, Theorem XII.2.5]. Further, $Q_{R}$ is flat by the result of Goodearl mentioned above (cf. also [5, Theorem 5.17] and [11, Theorem XII.7.1]). But then it follows from [11, Corollary XII.7.3] that $Q$ is also the maximal left quotient ring of $R$.

(iii) $\Rightarrow$ (i) Since $Q$ is semisimple, $R$ is right nonsingular by [11, Proposition XII.2.2]. Also, since the left maximal quotient ring $Q$ of $R$ is semisimple, the canonical homomorphism $R \rightarrow Q$ is a left flat epimorphism. Then, using again [5, Theorem 5.17 , we see that every finitely generated nonsingular right $R$-module embeds in a free module.

Finally, observe that the proof can be completed by symmetry, bearing in mind that condition (iii) is left-right symmetric.

An entirely similar argument can be applied to the characterization given by Cateforis and Goodearl of the right nonsingular rings such that every finitely generated nonsingular right $R$-module is projective [5, Theorem 5.18]. This class of rings is not right-left symmetric in general [5] but, from the preceding corollary and [5, Theorem 5.18], we have:

Corollary 2.6. Let $R$ be a ring such that r. $\operatorname{pgldim}(R) \leq 1$ and $Q$ its maximal right quotient ring. Then the following conditions are equivalent:

(i) $R$ is right nonsingular and every finitely generated nonsingular right $R$-module is projective.

(ii) $R$ is left nonsingular and every finitely generated nonsingular left $R$-module is projective.

(iii) $R$ is left and right semihereditary, and $Q$ is a semisimple two-sided maximal quotient ring of $R$.

\section{REFERENCES}

[1] R. R. Colby and E. A. Rutter, Jr., Generalizations of QF-3 algebras, Trans. Amer. Math. Soc. 153 (1971), 371-386. MR 42:4581

[2] C. Faith, Embedding modules in projectives, Lecture Notes in Math., vol. 951, Springer-Verlag, New York, 1982 pp. 21-39. MR 84i:16001 
[3] J. L. Gómez Pardo, Nguyen V. Dung, and R. Wisbauer, Complete pure injectivity and endomorphism rings, Proc. Amer. Math. Soc. 118 (1993), 1029-1034. MR 93j:16003

[4] K. R. Goodearl, Embedding nonsingular modules in free modules, J. Pure Appl. Algebra 1 (1971), 275-279. MR 45:8675

[5] _ Ring theory, Marcel Dekker, New York, 1976. MR 55:2970

[6] L. Gruson and C. U. Jensen, Dimensions cohomologiques relieés aux foncteurs $\lim ^{(i)}$, Lecture Notes in Math., vol. 867, Springer-Verlag, Berlin and New York, 1981, 234-294. MR 83d:16026

[7] R. Kielpinski and D. Simson, On pure homological dimension, Bull. Acad. Polon. Sci. 23 (1975), 1-6. MR 53:10872

[8] B. L. Osofsky, Rings all of whose finitely generated modules are injective, Pacific J. Math. 14 (1964), 645-650. MR 28:5090

[9] , Noninjective cyclic modules, Proc. Amer. Math. Soc. 19 (1968), 1383-1384. MR 38: 185

[10] E. A. Rutter, Jr., QF-3 rings with ascending chain condition on annihilators, J. Reine Angew. Math. 277 (1975), 40-44. MR 53:520

[11] B. Stenström, Rings of quotients, Springer-Verlag, Berlin and New York, 1975.

[12] B. Zimmermann-Huisgen and W. Zimmermann, Algebraically compact rings and modules, Math. Z. 161 (1978), 81-93. MR 58:16792

Departamento de Alxebra, Universidade de Santiago, 15771 Santiago de Compostela, SPAIN

E-mail address: pardo@zmat.usc.es

Departamento de Matematicas, Universidad de Murcia, 30100 Espinardo, Murcia, SPAIN

E-mail address: paguil@fcu.um.es 